\title{
The Population Response of A- and C-Fiber Nociceptors in Monkey Encodes High-Intensity Mechanical Stimuli
}

\author{
Robert M. Slugg, ${ }^{1}$ James N. Campbell, ${ }^{1,2}$ and Richard A. Meyer ${ }^{1,2}$ \\ ${ }^{1}$ Department of Neurosurgery and ${ }^{2}$ The Applied Physics Laboratory, Johns Hopkins University, Baltimore, Maryland 21287
}

\begin{abstract}
The peripheral neural mechanism of pain to mechanical stimuli remains elusive. C-fiber nociceptors do not appear to play a major role in mechanical pain sensation, because the stimulus-response function of mechanically sensitive C-fiber nociceptors to punctate mechanical stimuli applied to the most sensitive region in the receptive field (the hot spot) reaches a plateau at force levels insufficient to produce pain in humans. However, studies at the hot spot give an incomplete understanding of the inputs of nociceptors to the spinal cord. To estimate how the population of nociceptors responds to a punctate stimulus, it is necessary to know how the response varies with the position within the receptive field. For A-fiber and C-fiber nociceptors, we systemically measured the response to a $100 \mu \mathrm{m}$ wide blade stimulus as a function of position in the receptive field at different force levels. Highly reproducible receptive field response maps that contained multiple peaks and valleys were obtained. Some peaks were only $100 \mu \mathrm{m}$ wide. As force increased, the response and width of the peaks increased, the response in valleys increased, and new peaks appeared. The averaged response across the map provides an estimate of the population response and was found to increase monotonically with force over a large stimulus range for both A-fiber and C-fiber nociceptors. These data provide evidence that both C-fiber and A-fiber nociceptors may encode high-intensity mechanical stimuli.
\end{abstract}

Key words: afferent; C-fiber; cutaneous; mechanosensory; nociception; pain

\section{Introduction}

Pain is unique among sensory systems in that this sensation may be aroused by multiple energy modalities. Accordingly, chemical, thermal, and mechanical stimuli may all evoke pain when applied to the skin. The conventional nociceptor studied in primates (including humans) and other species responds to each of these stimulus modalities. Much progress has been made with regard to understanding pain evoked by heat (Raja et al., 1999). Responses to heat of nociceptors correlate highly with psychophysical ratings of pain. In addition, much progress has been made with regard to understanding the molecular basis of heat responses of nociceptors (Julius and Basbaum, 2001).

However, how pain to mechanical stimulation is encoded remains less clear. The responses of conventional C-fiber mechanically sensitive nociceptors in primate, for example, saturate at stimulus intensities well below the pain threshold in humans (Greenspan and McGillis, 1991; Garell et al., 1996; Slugg et al., 2000). Likewise, there is a poor correlation between the responses of conventional nociceptors and pain felt during both sustained mechanical stimuli and repeated impact stimuli (Koltzenburg and Handwerker, 1994; Schmidt et al., 2000).

Receptive field maps of cutaneous nociceptors traditionally have been determined with hand-held von Frey probes. These

Received Feb. 27, 2004; revised April 8, 2004; accepted April 9, 2004.

This research was supported by National Institutes of Health Grants NS14447 and NS09260. We thank Dr. Matthias Ringkamp for helpful discussions and Timothy Hartke and Sylvia Horasek for their technical assistance.

Correspondence should be addressed to Richard A. Meyer, Department of Neurosurgery, Meyer Building, Room 5-109, Johns Hopkins University School of Medicine, 600 North Wolfe Street, Baltimore, MD 21287. E-mail: rmeyer@jhmi.edu.

DOI:10.1523/JNEUROSCI.0701-04.2004

Copyright $\odot 2004$ Society for Neuroscience $\quad$ 0270-6474/04/244649-08\$15.00/0 studies reveal that the receptive fields have discrete regions of high sensitivity (hot spots). When a given region of skin is stimulated with a punctate stimulus, most nociceptors are activated in a region remote from their hot spots. To obtain an estimate of nociceptor response in aggregate to a punctate mechanical stimulus, it is necessary to determine how a given nociceptor responds to stimuli presented at various positions within its receptive field. Although a saturation of response may occur for stimuli applied to the hot spots, we postulated that the population response of nociceptors may not saturate at higher forces, because areas with low sensitivity may still grow in responsiveness as a function of stimulus intensity.

To map the responsiveness of nociceptors, we developed a computer-controlled mechanical stimulator system that allows a forcecontrolled stimulus to be accurately applied to different locations on the skin (Schneider et al., 1995). Previous research suggests that nociceptors respond to stresses at the edges rather than simple indentation (Greenspan and McGillis, 1991) and that $100 \mu \mathrm{m}$ resolution is needed to resolve nociceptor hot spots (Schneider et al., 1995). To get around the daunting task of mapping the response to a punctate stimulus (requiring $>10,000$ stimuli to obtain $100 \mu \mathrm{m}$ resolution in a $1 \mathrm{~cm}^{2}$ area), we elected to use a $100 \mu \mathrm{m}$ thick blade stimulus that extended across the width of the receptive field. This approach collapses the receptive field $x-y$ coordinates to a single dimension.

We obtained functional receptive field maps at different forces for A- and C-fiber nociceptors. The population response functions from these nociceptors did not exhibit saturation at the higher force levels.

\section{Materials and Methods}

Neurophysiological preparation

A standard teased-fiber technique (Campbell and Meyer, 1983) was used to record from single primary afferents in cutaneous, peripheral nerves in 
monkeys (Macaca fascicularis; 5-7 kg). Monkeys were sedated with ketamine and then anesthetized to a level such that the corneal reflex was absent by intravenous administration of a mixture of sodium pentobarbital $(3 \mathrm{mg} / \mathrm{kg} / \mathrm{hr})$ and morphine sulfate $(0.5 \mathrm{mg} / \mathrm{kg} / \mathrm{hr})$. Animals were intubated, and peak expired $\mathrm{pCO}_{2}$ was maintained at 35-40 torr using mechanical ventilation. The animals were paralyzed with pancuronium bromide $(0.1 \mathrm{mg} / \mathrm{kg})$ to minimize muscle artifacts during recordings as well as to facilitate respiratory control. The rectal temperature was controlled at $38^{\circ} \mathrm{C}$ by means of a circulating water heating pad. Five percent dextrose in $0.9 \%$ normal saline was administered intravenously in the course of the experiments to maintain hydration. Adequate depth of anesthesia was ensured by continuous monitoring of the heart with an electrocardiogram. The heart rate was maintained throughout the experiment within $10 \%$ of the baseline heart rate before any surgical stimulus. Any sudden increase in heart rate that was temporally related to a surgical or test stimulus was treated with an additional bolus of the intravenous anesthetics. When it became apparent that the animal was spontaneously breathing $(\sim 2-3 \mathrm{hr}$ after pancuronium bromide administration), the absence of motor responses to noxious stimuli was verified, and an additional bolus dose of pancuronium bromide was administered. All protocols were approved by the University Animal Care and Use Committee. Animal housing conforms to federal regulations, and the facilities are accredited by the American Association for Accreditation of Laboratory Animal Care.

An incision was made in the skin over the cutaneous nerve of interest, and the edges of the incision were tied to a ring to form a well into which paraffin oil was placed. Small bundles of axons were cut away from the nerve trunk and dissected to fine strands that were placed on the recording electrode. The strands from which recordings were made were cut proximally so that only centripetally conducted action potentials (APs) were recorded.

Receptive fields were located by gently squeezing the skin in the distribution of the nerve under study. Calibrated nylon monofilaments (von Frey type, Stoelting aesthesiometer set; Stoelting, Wood Dale, IL) were used to locate sensitive spots in the receptive field and to determine their response thresholds. The boundary of the receptive field was determined with a suprathreshold von Frey filament. All receptive fields were located on the distal hairy skin of either the upper or lower extremity.

Fibers were classified as nociceptors if they responded to pinching of the skin but did not respond to blunt pressure or light stroking. No attempt was made to subclassify A-fibers into type I and type II A-fiber mechano-heat-sensitive nociceptors (Treede et al., 1998) to avoid thermally induced sensitization. Nociceptors were studied only if their von Frey threshold was not $<5 \mathrm{mN}$ to eliminate the possible inclusion of low-threshold slowly adapting mechanoreceptors in the study.

\section{Mechanical stimulation protocols}

Mechanical stimulator. A computer-based electromechanical stimulator system was used to apply controlled force stimuli to different locations within the receptive field (Schneider et al., 1995). The core of the stimulator is a servo-controlled linear motor capable of generating $10 \mathrm{~N}$ of force over a $22 \mathrm{~mm}$ range. Forces that are collinear and tangential to the interchangeable probe tip are calculated using the signal from three load cells ( $1 \mathrm{mN}$ resolution, $2.5 \mathrm{~N}$ range) arranged in an equilateral triangle. Probe position is measured with an optical encoder ( $1 \mu \mathrm{m}$ resolution, 25 $\mathrm{mm}$ range). A microprocessor-based digital control system allows feedback control to be switched smoothly between force and position at the 1 $\mathrm{KHz}$ update rate. The stimulator is mounted on a microprocessorcontrolled three-axis translation system that allows automatic move-
B

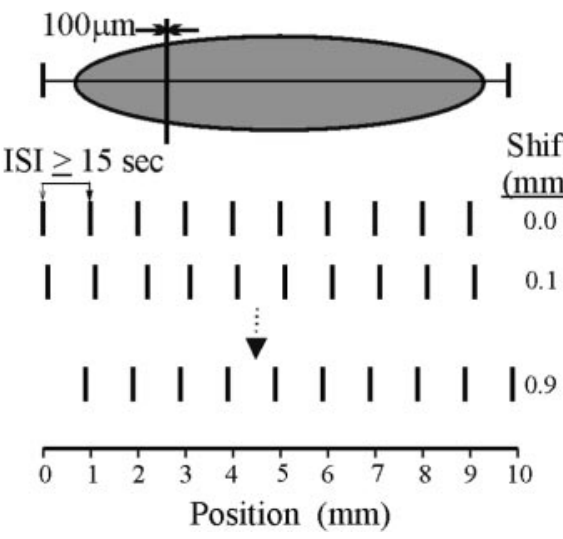
0.1 0.9

Time $(\mathrm{sec})$ $\mu \mathrm{m}$. The stimulator can be programmed to move in a coordinate system parallel to the skin surface being examined. An IBM-compatible computer is used to command stimulus paradigms and to display real-time motor performance and neural spike-train data.

Stimulus waveform. A typical stimulus waveform is shown in Figure $1 \mathrm{~A}$. Each stimulus trial started with a $4 \mathrm{sec}$ prestimulus period during which the probe was moved under position control from a position $\sim 1$ $\mathrm{mm}$ above the skin surface to a position at which skin contact was made as detected by an increase in the measured probe force. At this point, the stimulator control automatically switched to force control, and a $5 \mathrm{mN}$ resting force was applied for the remaining time in the prestimulus period. The $1 \mathrm{sec}$ stimulus period consisted of a $0.2 \mathrm{sec}$ force-controlled ramp to the desired force and a $0.8 \mathrm{sec}$ constant force interval. At the end of the stimulus, the probe was withdrawn in position-control mode to a position $1 \mathrm{~mm}$ above the skin surface. During the intertrial interval, the stimulator was automatically moved to the next position in the receptive field as determined by the mapping protocol (see below).

Stimulus interaction experiments. In previous experiments, we observed a fatigue in the response when mechanical stimuli were present at the same location in the receptive field of nociceptors. The time for full recovery from fatigue was $\sim 5 \mathrm{~min}$ for the $\mathrm{C}$-fiber nociceptors and $<1$ min for the A-fiber nociceptors (Slugg et al., 2000). Because we wished to minimize fatigue that might occur during the mapping protocol, we determined whether fatigue also occurs when a conditioning stimulus is applied at a distant spot in the receptive field. Two sites that were $1 \mathrm{~mm}$ apart and had approximately equal responsiveness to mechanical stimuli were investigated (see Fig. 2A).

The timing of the stimuli at each site is illustrated in Figure $2 B$. Site $B$ was the test site, and site A was the conditioning site. The first test stimulus was presented after at least a $10 \mathrm{~min}$ stimulus-free interval, and the response to this stimulus was considered to be fully recovered from any fatigue. The subsequent test stimuli followed a conditioning stimulus at interstimulus intervals (ISIs) of 300, 150, 60, 30, or $15 \mathrm{sec}$ (presented in descending order). The conditioning stimulus always occurred $10 \mathrm{~min}$ after the last test stimulus to ensure full recovery.

Mapping protocol. Force-controlled stimuli (40-640 $\mathrm{mN}$ in factor of two increments, $1 \mathrm{sec}$ duration) were delivered to the skin with the mechanical stimulator via thin $(100 \mu \mathrm{m})$ blade-shaped probes. Bladeshaped probes were chosen to permit high spatial resolution maps in one 
Table 1. Average conduction velocities, von Frey thresholds, and receptive field areas determined with von Frey probes

\begin{tabular}{lll}
\hline & A-fiber nociceptors & C-fiber nociceptors \\
\hline Number of fibers & 32 & 27 \\
Conduction velocity $(\mathrm{m} / \mathrm{sec})$ & $33 \pm 3$ & $0.82 \pm 0.05$ \\
Receptive field area $\left(\mathrm{mm}^{2}\right)$ & $49 \pm 8^{* *}$ & $25 \pm 3$ \\
von Frey threshold (bar) & $3.9 \pm 0.3$ & $3.6 \pm 0.4$ \\
\hline
\end{tabular}

${ }^{* *} p \leq 0.01 ;$ t test.

dimension (perpendicular to the blade) while simultaneously activating mechanically responsive endings in the receptive field in the orthogonal dimension (along the length of the blade). Three different blade shapes were used: a $0.1 \mu \mathrm{m}$ wide, $10 \mu \mathrm{m}$ long rectangular blade; a $0.1 \mu \mathrm{m}$ wide, $8 \mu \mathrm{m}$ diameter circular blade; and a $0.1 \mu \mathrm{m}$ wide, $8 \mu \mathrm{m}$ diameter circular blade in which the bottom was slightly flattened. The rectangular blade was used when the receptive field was located on round structures, such as a finger, and the ends of the blade extended off the skin. The other blades were used for receptive fields on flat surfaces and were rounded at the ends to avoid stress concentration in the tissue that would occur if the blades had square corners.

Maps of the receptive field structure in one dimension were obtained by applying the blade probe at $0.1 \mathrm{~mm}$ increments along a line through the center of the receptive field parallel to the long axis (Fig. $1 B$ ). This strategy permits functional mapping of two-dimensional structures from data obtained in one dimension. As an example, a $10 \mathrm{~mm}$ long map at 0.1 $\mathrm{mm}$ increments required 100 stimulus trials (Fig. $1 \mathrm{~B}$ ). Because immediately adjacent sites shared a common edge of the stimulus probe, the time constant for recovery from fatigue may be comparable with that for stimuli presented to the same site (Slugg et al., 2000). To minimize both mapping time and stimulus interaction effects, stimuli were applied sequentially at $1 \mathrm{~mm}$ increments (i.e., positions $0,1.0,2.0,3.0,4.0,5.0,6.0$, $7.0,8.0$, and 9.0). The time between stimuli was $15 \mathrm{sec}$ for A-fibers and 30 sec for C-fibers. After 10 stimuli, the sequence was shifted by $0.1 \mathrm{~mm}$, and the $1 \mathrm{~mm}$ increments were repeated (i.e., positions $0.1,1.1,2.1,3.1,4.1$, 5.1, 6.1, 7.1, 8.1, and 9.1). This process was continued until all positions were stimulated. Thus, for a 100-point map, the time between stimulation at adjacent positions was $150 \mathrm{sec}$ for A-fibers and $300 \mathrm{sec}$ for C-fibers. A 100-point map required $\sim 25$ min for an A-fiber nociceptor (100 points at $15 \mathrm{sec}$ each) and $50 \mathrm{~min}$ for a C-fiber nociceptor (100 points at $30 \mathrm{sec}$ each). For maps that were shorter than 100 points, the interstimulus interval was adjusted so that the stimuli that were applied to immediately adjacent spots were $>1 \mathrm{~min}$ apart for A-fibers and $5 \mathrm{~min}$ apart for C-fibers.

Stimulus-response functions. The force levels used during mapping were multiples of two ranging from 40 to $640 \mathrm{mN}$. In general, the force used during the first map of the receptive field was chosen to be just suprathreshold to the force threshold of the fiber determined with von Frey probes. Thus, fibers with low thresholds started with lower force levels than fibers with higher thresholds. After completing the first map, subsequent maps were obtained at different force levels. For most fibers, a family of maps was obtained at different force levels.

\section{Results}

Teased-fiber techniques were used to record from $32 \mathrm{~A}$-fiber and $27 \mathrm{C}$-fiber nociceptors in the anesthetized monkey. The average conduction velocities, von Frey thresholds, and receptive field areas, as determined with von Frey probes, are summarized in Table 1.

\section{Stimulus interaction from spatially separated stimuli}

Nociceptors exhibit fatigue (desensitization) in response to repeated mechanical stimuli applied to the same location (Slugg et al., 2000). For C-fiber nociceptors, full recovery from this fatigue can take up to $5 \mathrm{~min}$. To map the receptive field, multiple stimuli are delivered to different locations in the receptive field. To verify that fatigue does not occur during our mapping protocol, we
A
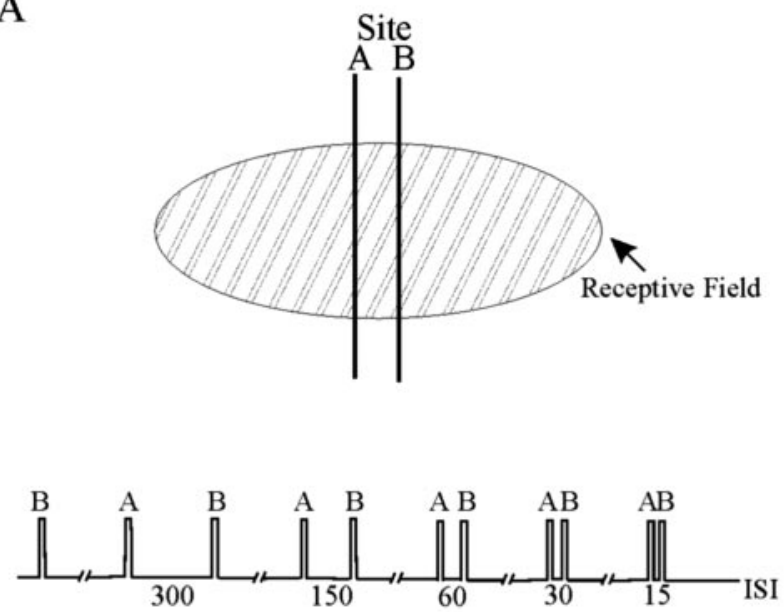

B
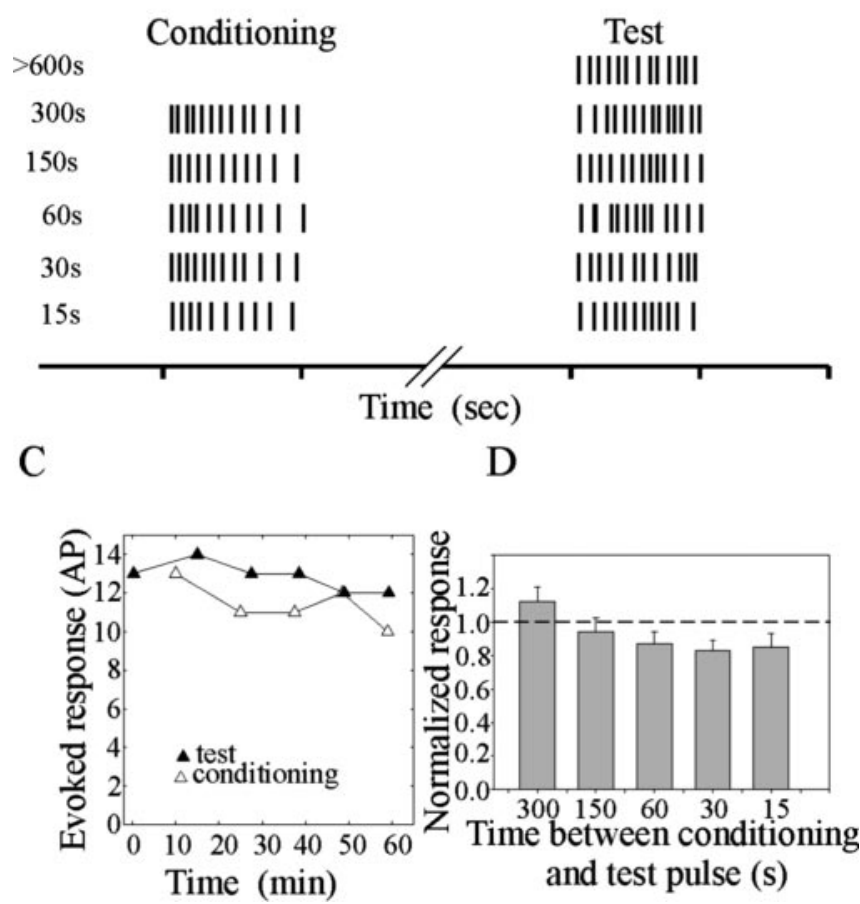

Figure 2. Stimulus interaction for spatially separated stimuli. A, Stimulus interaction protocol. Top, The test site (site B) and the conditioning site (site A) were $1 \mathrm{~mm}$ apart. Bottom, Time line for the conditioning and test stimuli. B, Response of a typical C-fiber nociceptor to the stimulus interaction paradigm. Each row corresponds to one conditioning test pair with the time between stimuli indicated in the left column. The vertical lines represent the action potential spikes evoked from the $1 \mathrm{sec}$ duration $80 \mathrm{mN}$ stimulus. C, The total response during the stimulus is plotted as a function of time for the test stimulus (filled triangles) and the conditioning stimulus (open triangles) for this typical C-fiber. D, Average response to the test stimulus as a function of time between the conditioning and test stimulus for $13 \mathrm{C}$-fiber nociceptors. For each fiber, the response for a given interstimulus interval was normalized by dividing by the response of that fiber to the first stimulus delivered to the test site.

tested the response to stimuli presented at one location in the receptive field after a conditioning stimulus was delivered at a second responsive location.

The response of a typical C-fiber is shown in Figure 2, $B$ and $C$. The response at the conditioning site decreased slightly with time, which we attribute to the fatigue resulting from repeated stimulation at a given site (Fig. 2C). A similar decrease was observed at the test site. No additional fatigue at the test site was obvious as a result of the conditioning stimulus even for the $15 \mathrm{sec}$ ISI. 
The average response of the population of C-fibers $(n=13)$ tested with this paradigm tended to decrease as the ISI decreased (Fig. 2D). A $15 \pm 8 \%$ decrease was seen for the $15 \mathrm{sec}$ ISI. Thus, we conclude that an ISI of $30 \mathrm{sec}$ will not lead to substantial fatigue effects during our mapping protocol if sequential sites are $\geq 1 \mathrm{~mm}$ apart. However, immediately adjacent stimulus sites (e.g., positions $0.0,0.1$, etc.) share a common edge and may be subject to stimulus interaction similar to that found for stimuli applied to a single spot (Slugg et al., 2000). Therefore, mapping protocols were altered as necessary so that stimulation at adjacent sites was separated by a minimum of $5 \mathrm{~min}$ for C-fibers and 1 min for A-fibers.

\section{Reproducibility of receptive field response maps}

In 22 fibers, the receptive field response map at a given force was repeated to test the reproducibility of the map. Examples of repeated maps in an A-fiber and a C-fiber nociceptor are shown in Figure 3, $A$ and $D$. The receptive field response maps of each fiber show multiple peaks and valleys. This structure was reproduced in a second map of each of these fibers. As a measure of the reproducibility of the maps, we created a difference map in which the response at each position in the second map was subtracted from the response at that position in the first map (Fig. $3 B, E$ ). Some variability occurred across the receptive field with the greatest variability occurring near the peak response. Because the general shape of the receptive field response maps did not change, we think this variability could be attributable to slight movement of the preparation between maps. The distribution of the differences is shown in Figure 3, $C$ and F. A majority of sites for the A-fiber map (51 of 93 ) and the C-fiber map (38 of 72) had a response within $\pm 1 \mathrm{AP}$ for the two maps. The SD of this difference distribution was 1.87 APs for the A-fiber and 2.14 APs for the C-fiber. The average SD of the difference distribution for all fibers in which at least two receptive field response maps at a given force were obtained was $1.8 \pm 0.2$ APs for the A-fibers $(n=15)$ and $2.3 \pm 0.3$ APs for the C-fibers $(n=7)$. There was no significant change in the total response for either the A-fibers or the $\mathrm{C}$-fibers, and thus the mapping procedure did not lead to sensitization or lasting desensitization. It is apparent that this procedure yields reproducible maps of the receptive field response structure.

\section{Comparison of the receptive field response map obtained with the blade probe to the map obtained with a von Frey probe}

Many of the features that are apparent in a conventional von Frey map of the receptive field were observed in the receptive field response maps obtained with the blade probe. For example, the receptive field response map for a typical C-fiber nociceptor is shown in Figure $4 A$. For this fiber, 11 spots with high sensitivity to a von Frey probe were identified (Fig. 4, inset). The map obtained with the blade probe shows multiple peaks, some of which
A-fiber Nociceptor

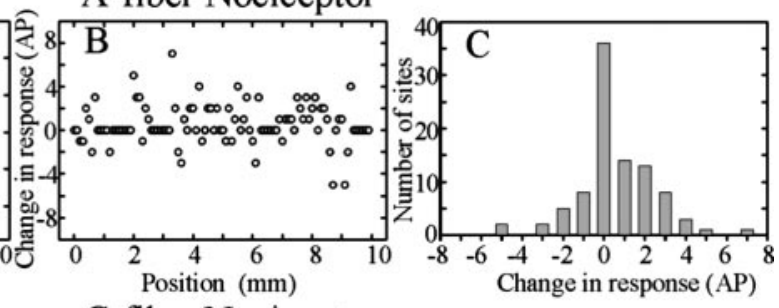

ล C-fiber Nociceptor

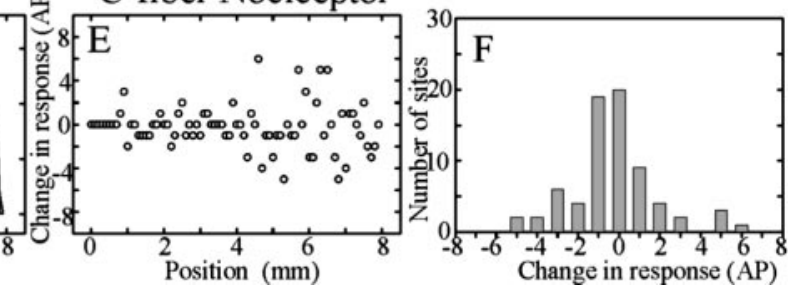

Change in response (AP)

Figure 3. Reproducibility of receptive field response maps. Top, Typical A-fiber nociceptor. Bottom, Typical C-fiber nociceptor. Receptive field response map for an A-fiber nociceptor (11 mN threshold; $38 \mathrm{~m} / \mathrm{sec}$ conduction velocity) located on the middle of the fourth finger. The mapping protocol described in Figure 1 was used. After a 10 min recovery period, a second map Distribution of differences for the C-fiber nociceptor. For the majority of sites, the response in the second map was within $\pm 1 \mathrm{AP}$

corresponded to the sensitive spots that were located with the von Frey probes. Several of these peaks are quite narrow, suggesting that the sensitive region was small.

The length of the receptive field obtained from the receptive field response map was estimated as the total interval in the map over which a response of at least one action potential was recorded at the highest force. This length correlated closely with the length of the receptive field obtained from measurements with the von Frey probes (Fig. $4 B$ ).

The threshold of the most sensitive spot in the receptive field was measured with the von Frey probes. Threshold was not directly measured with the blade probe. Instead, we determined the peak response across the receptive field response map obtained at a $160 \mathrm{mN}$ force. This peak response was inversely correlated to the von Frey threshold (Fig. 4C).

\section{Structure in receptive field response maps}

Three general types of receptive field architecture were observed in the receptive field response maps: (1) multiple, fine structures with narrow peaks sometimes being only one probe position wide (Figs. 3A, 4A, 5A); (2) broad, rounded structures (Fig. 5B); and (3) punctate structures superimposed on a broad baseline structure (Figs. 3D, 5C, 5D). These three different types of structures were observed in both A-fiber and C-fiber nociceptors. The receptive field map architecture was not a property of skin location or compliance. The fine structure in these maps demonstrate that the area of peak responsiveness can be as small as the width of the blade stimulus, which was $100 \mu \mathrm{m}$. For a given receptive field, the size and shape of the different peaks could vary. Some peaks gave a much bigger response then others. Some peaks were narrow, whereas others were broader. 
A

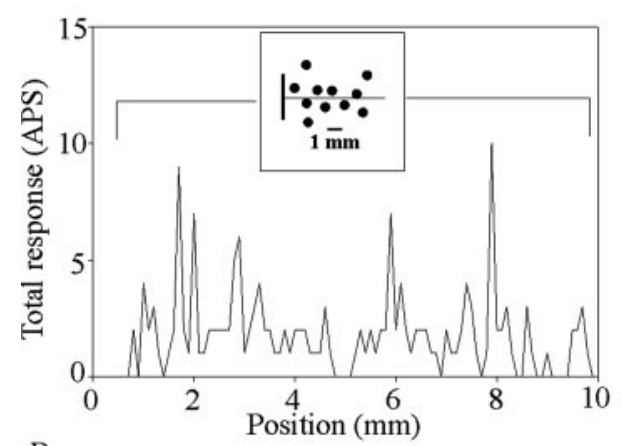

B
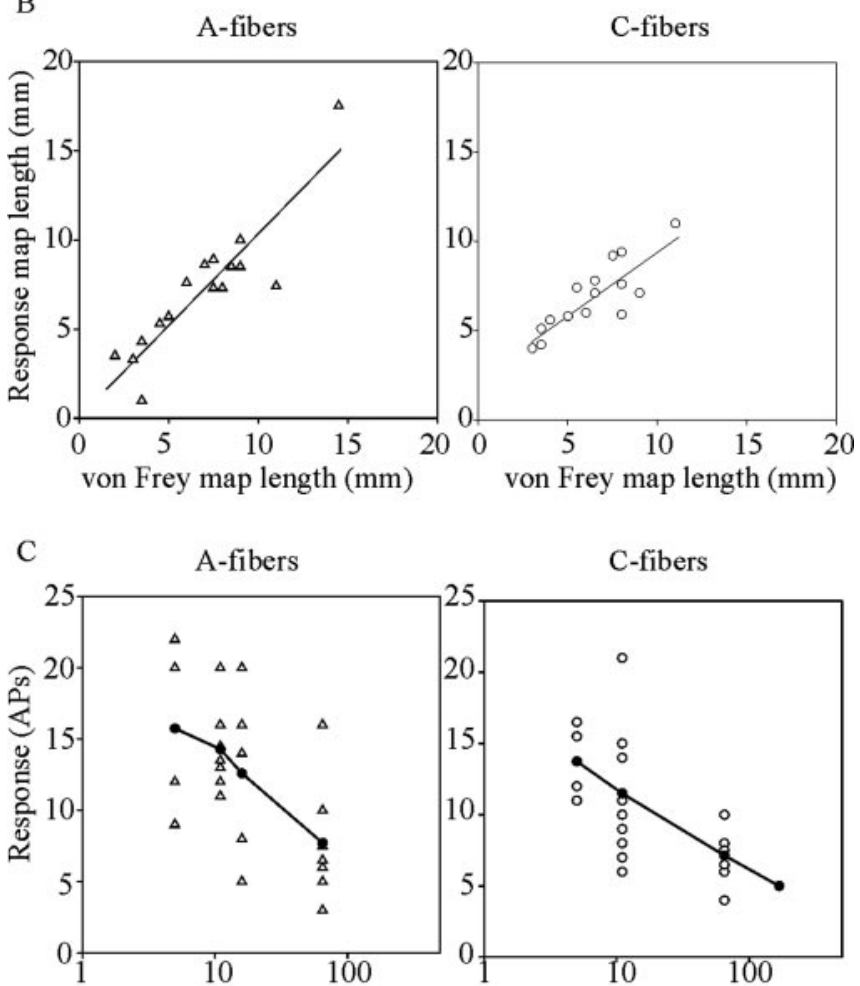

von Frey threshold $(\mathrm{mN})$

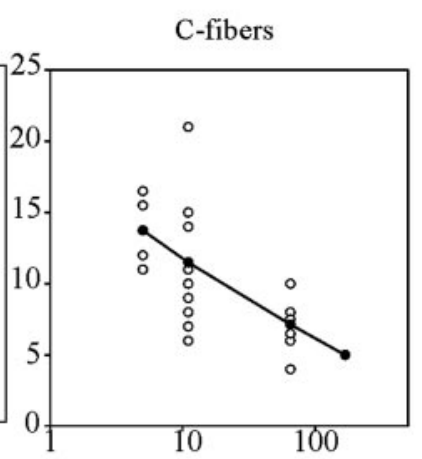

von Frey threshold $(\mathrm{mN})$

Figure 4. Comparison of data obtained with a von Frey probe and with the blade-shaped probe. $A$, Receptive field response maps. The mapping protocol described in Figure 1 was used to obtain a response map for a C-fiber nociceptor ( $11 \mathrm{mN}$ threshold; $0.7 \mathrm{~m} / \mathrm{sec}$ conduction velocity) with a receptive field on the lateral volar forearm. The total response of the C-fiber to a $160 \mathrm{mN}, 1$ sec duration controlled force stimulus is plotted as a function of probe position. This receptive field response map revealed a number of peaks, some of which are quite narrow. Inset, The receptive field structure obtained with a $65 \mathrm{mN}$ von Frey probe revealed 11 sensitive spots. Some of the peaks in the receptive field response map correspond to the sensitive spots obtained with the von Frey probe. Note that two of the spots in the von Frey map were outside the width of the blade probe that was used and thus were not likely stimulated by the probe. $B$, Length of receptive field. For each fiber, the length of the receptive field determined from the blade probe is plotted versus the length along the mapping axis as determined by the von Frey probe. Left, A-fibers $(n=19)$. Right, C-fibers $(n=15)$. C, Magnitude of response. For each fiber, the maximum response in the receptive field to a $160 \mathrm{mN}$ stimulus from the blade probe is plotted versus the von Frey threshold for that fiber. Left, A-fibers $(n=23)$. Right, $(-$ fibers $(n=22)$.

\section{Stimulus response functions}

For 16 A-fiber nociceptors and 20 C-fiber nociceptors, receptive field maps were obtained at two or more force levels. Examples from four different fibers are shown in Figure 5. Although the magnitude of the response increases as the force increases, the general structure of these maps remains relatively stable. The peaks grow in height and width, the valleys between the peaks become less distinct,

and some areas that were unresponsive at low forces become responsive at higher forces. In some cases, new peaks emerge as the force increases.

The stimulus response function at different points in the receptive field of these four fibers is shown in the corresponding middle panels in Figure 5. For a given fiber, the shape of the stimulus-response functions varies substantially from point to point. Some locations show a monotonically increasing response, whereas others demonstrate saturation in the stimulus-response function.

\section{Averaged response functions}

When a mechanical stimulus is applied to the skin, a population of nociceptive afferents is activated. For some of these afferents, the stimulus is located on a sensitive region, whereas for others, the stimulus is in a less sensitive but nevertheless responsive area. Because we cannot measure the response of all afferents that innervate the stimulus location, one approach to estimating the population response to a given stimulus is to measure the response to that stimulus throughout the receptive field of a given afferent and assume that this afferent represents well the properties of a typical afferent. The average of the responses across the receptive field then provides an estimate of the population response to the stimulus.

The receptive field response maps described above provide a means to estimate the population response of the nociceptors. The average response was computed over all positions in the receptive field (excluding zero values at either end). The averaged response for the four nociceptors in Figure 5 is given in the bottom panels of Figure 5. The averaged response for each fiber increased monotonically with stimulus force over the full range of stimulus intensities.

The averaged response for all fibers in this study for which a receptive field response map was obtained at two or more forces is shown in Figure 6. It should be noted that the averaged response across the receptive field for each fiber exhibited a monotonically increasing response with increasing force over the full stimulus range investigated. This was true even for fibers studied at five different stimulus intensities representing a 16-fold increase in force.

Although the magnitude of the averaged responses for the A-fibers and C-fibers in this study was similar (Fig. 6), the stimulus response functions for the A-fibers were steeper than for the C-fibers. To estimate how fast the response grew with increasing force, we computed the ratio of the response to successive forces (i.e., the response to one force was divided by the response to the next lower force). The response of the A-fibers approximately quadruples for every doubling of force, whereas the response of the C-fibers approximately doubles (Fig. 7).

\section{Discussion}

This is the first study that systematically measured the responses of primate nociceptors to mechanical stimuli as a function of stimulus intensity and of position within the receptive field. We demonstrate that receptive field response maps contain both reproducible structure and response. These reproducible maps will be useful for studying the role of mechanical sensitization of nociceptors as a mechanism for primary mechanical hyperalgesia.

\section{Stimulus interaction effects}

Strong temporal interaction occurs in nociceptors (Peng et al., 2003). When a mechanical stimulus is applied to the same location in the receptive field at an interstimulus interval of $30 \mathrm{sec}$, the 
response to the second stimulus is $80 \%$ of the response to the first stimulus for A-fibers and 70\% for C-fibers (Slugg et al., 2000). The recovery time is $\sim 1$ min for A-fibers and $5 \mathrm{~min}$ for C-fibers.

To obtain an accurate map of mechanical responsiveness, we needed to minimize stimulus interaction effects but also minimize the time required to complete the map. Homologous desensitization refers to the decrement in response that occurs when the test and conditioning stimulus are of the same modality and are applied to the same location. Heterologous desensitization refers to the change in response when the conditioning stimulus is of a different modality or when it is delivered to a different location within the receptive field. For heat-test stimuli, the time constant for recovery of C-fibers from heterologous desensitization $(\approx 30$ sec) (Peng et al., 2003) is shorter than from homologous desensitization $(>2 \mathrm{~min})$ (Tillman, 1992). We determined that stimulus interaction was not significant for mechanical stimuli applied $1 \mathrm{~mm}$ apart at a $60 \mathrm{sec}$ interstimulus interval. Thus, as with heat stimuli, heterologous desensitization has a shorter duration. Heterologous desensitization is likely attributable to the antidromic invasion of action potentials from the conditioning site to the test site. With homologous desensitization, the conditioning stimulus engages the transduction mechanism, which likely has a long recovery cycle.

\section{Structure of receptive field response maps}

Inspection of the receptive field response maps reveals several salient findings. First, the structure of the maps is complex with many peaks and valleys of different dimensions. High responsiveness in one region does not necessarily predict high responsiveness in another. Second, some maps were dominated by sharp peaks (as narrow as the $100 \mu \mathrm{m}$ sampling interval), whereas others contained broad peaks. This was true for both A- and C-fiber nociceptors. There was no obvious relationship of sharpness of the maps with other characteristics such as receptive field size and mechanical thresholds. Third, the shape of the map was highly reproducible for sequential maps. Fourth, the height and shape of the peaks varied considerably within the receptive area of a given nociceptor. Finally, although there was considerable inter-fiber variability, A- and Cfibers had similar maps overall.

There are several potential explanations for the different shaped peaks. First, deeper terminals may have broader peaks because of stimulus spread. Superficial terminals would be more sensitive to small changes in stimulus position leading to sharper peaks. Supporting this hypothesis is the finding that heatfiber.
A-fiber Nociceptors
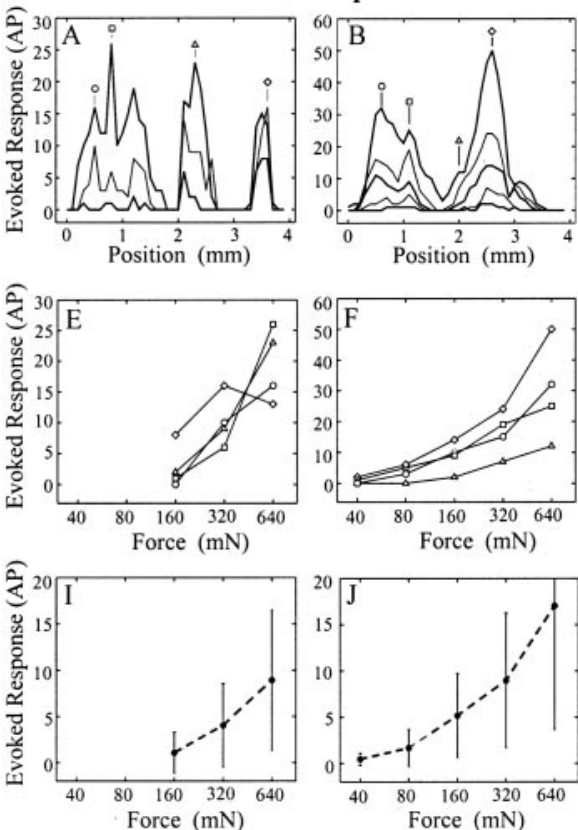

C-fiber Nociceptors
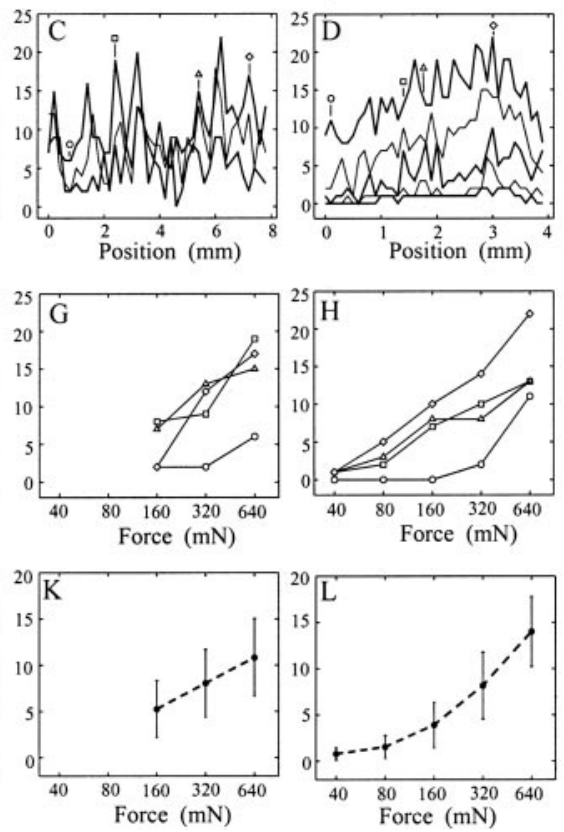

Figure 5. Responses of two A- and two C-fiber nociceptors at different stimulus intensities. Top, Receptive field response maps. Middle, Stimulus response functions at specific locations (indicated by symbol) in the receptive field response map. Bottom, Averaged response across the receptive field. Left two columns, Typical A-fiber nociceptors. Right two columns, Typical C-fiber nociceptors. A, An A-fiber mechano-cold nociceptor (von Frey threshold, $11 \mathrm{mN}$ ) located on the middle phalanx of the fourth finger. Receptive field response maps were made at 160, 320, and $640 \mathrm{mN}$. B, An A-fiber nociceptor (von Frey threshold $65 \mathrm{mN}$ ) located on the knuckle of the third digit. Receptive field response maps were made at 40,80, 160,320, and $640 \mathrm{mN}$. C, A C-fiber mechano-heat nociceptor (von Frey threshold, $5 \mathrm{mN}$ ) located on the volar forearm near the wrist. Receptive field response maps were made at 160,320, and $640 \mathrm{mN}$. D, A C-fiber mechano-heat nociceptor (von Frey threshold $65 \mathrm{mN}$ ) located on the medial side of the foot. Receptive field response maps were made at 40, 80, 160, 320, and $640 \mathrm{mN}$. E-H, Corresponding stimulus response functions. For each fiber, the stimulus response functions at four sites in the receptive field are displayed. The symbol in the stimulus response function corresponds to the symbol at the site in the response map. $I-L$, The averaged response ( $\pm S D$ ) of all sites within the receptive field response map is plotted for each fiber.
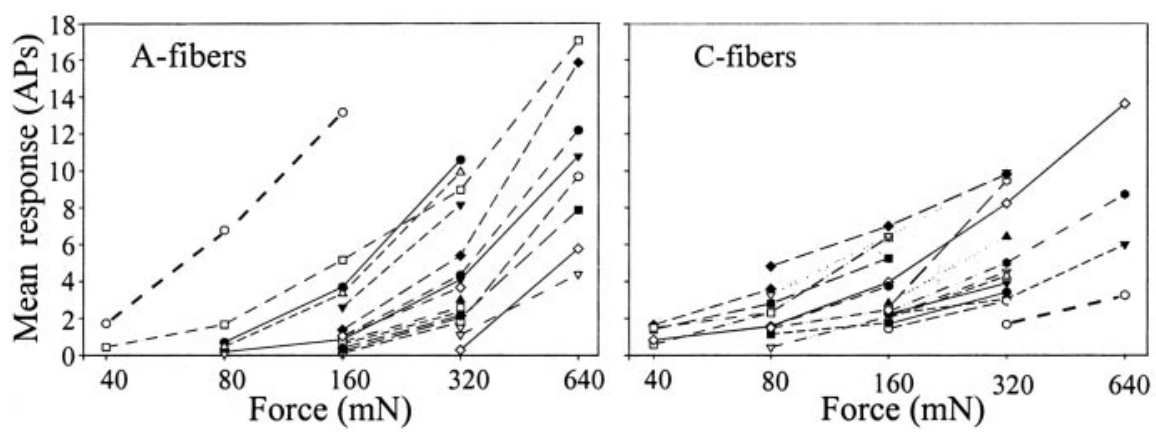

Figure 6. Averaged response functions increase monotonically with force. Left, A-fibers $(n=16)$. Right, $C$-fibers $(n=20)$. For each fiber, the average response across the receptive field for each force was determined. Each symbol corresponds to a different

sensitive terminals of C-fiber nociceptors are located at depths that range from 20 to $570 \mu \mathrm{m}$ (Tillman et al., 1995). Second, isolated terminals would lead to sharp peaks, whereas clusters of terminals would lead to broad peaks. Third, the orientation of the endings may influence shape. If transduction occurs over a length of the terminal (rather than just the ending), terminals that are orthogonal to the skin surface would produce sharp peaks, whereas terminals that run parallel to the skin surface (and orthogonal to the blade) would produce broad peaks. Fourth, given that the blade stimulus activates terminals across the width of the 


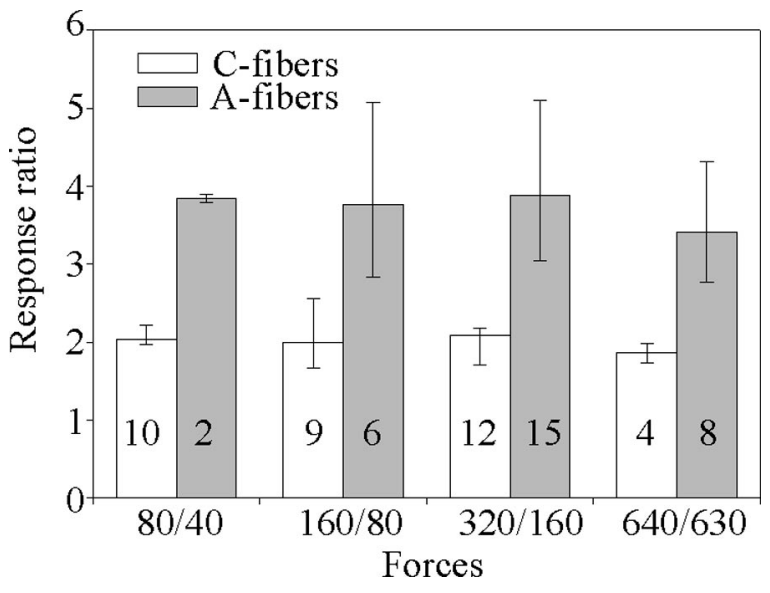

Figure 7. Growth of stimulus response function is greater for A-fibers than for C-fibers. The response of $A$-fibers quadruples for every doubling of force, whereas the response of $C$-fibers doubles. For each fiber, the averaged response at the higher force is divided by the averaged response at the lower force. Number of fibers from which the ratio was calculated is indicated within each bar.

receptive field, movement of the probe by $100 \mu \mathrm{m}$ may lead to activation of a terminal at a different location along the blade. Thus, receptive fields with a high density of receptive terminals would more likely have broad peaks, whereas receptive fields with a low density would have narrow peaks. Finally, terminals that are more responsive to shearing and tensile stresses may have broader peaks than those most responsive to compressive stresses.

Our finding that some receptive field response maps exhibit narrow peaks suggests that previous studies with hand-held probes may have missed the area of maximum sensitivity. Thus, estimates of the von Frey thresholds may be high, because a missalignment of as little as $100 \mu \mathrm{m}$ could place the probe away from the most sensitive spot.

In primate, systematic receptive field maps have only been obtained for low-threshold mechanoreceptors. Although the majority of work has been done in A-fiber mechanoreceptors (Johnson and Lamb, 1981; Vallbo et al., 1995), a recent study investigated C-fiber low-threshold mechanoreceptors in humans (Wessberg et al., 2003). These C-fibers were mapped with different forces using a $1 \mathrm{~mm}$ ball-tipped stroking stimulus that moved across the skin at $2 \mathrm{~mm} / \mathrm{sec}$. Although these fibers were clearly non-nociceptive (thresholds of $\sim 1 \mathrm{mN}$ ), their response maps have similarities with the maps shown here for nociceptive fibers with regard to number, width, and size of peaks. The dragging stimulus leads to stimulus interaction effects that alter the shapes of the peaks.

Others have mapped the responses of nociceptors in species other than primate. In rabbit, Kenins (1988) found that functional maps obtained with vibrating wool fibers became more complex as the diameter of the fibers decreased. Khalsa et al. (2000) used a cylindrical probe with a diameter $(3 \mathrm{~mm})$ comparable with the receptive field size of nociceptors in an in vitro rat skin preparation. These maps were smaller and less complex than those observed in the present study.

Stimulus-response functions and psychophysical correlations For many fibers, complete receptive field response maps were obtained at two or more force levels. As the force increased, the response at the peaks increased, the width of the peaks broad- ened, and new peaks developed. This implies that recruitment plays a role in accounting for increasing pain with increasing stimulus intensity.

The shape of the stimulus-response function varied with position in the receptive field (Fig. 5). For some locations, the response increased monotonically with force but in other locations, the response approached a plateau (or even had an inverted U-function) at the higher forces. In contrast, the average response across the receptive field always increased monotonically with force.

For most previous studies, the mechanical stimulus-response functions were obtained at the area of maximum sensitivity. At these hot spots, the stimulus-response functions of C-fiber nociceptors reached a plateau at force levels substantially below the pain threshold (Greenspan and McGillis, 1991; Khalsa et al., 1997; Andrew and Greenspan, 1999; Slugg et al., 2000). The flatness of the stimulus-response curves suggested that C-fiber nociceptors could not encode painfulness of punctate mechanical stimuli. One reconciliation of this paradox is that recruitment of mechanically insensitive nociceptors (nociceptors with mechanical thresholds more than six bars) may be necessary to explain pain to mechanical stimuli (Garell et al., 1996). The hypothesis advanced in this study is that pain ratings are related to the response properties of the population of nociceptors that are activated by the stimulus. This requires knowledge of how the response varies with stimulus position, because most of the nociceptors excited by a punctate stimulus would not be activated directly at a hot spot.

A standard approach to estimating the population response is to determine the response when the stimulus is placed at every possible location in the receptive field. The population response is estimated by averaging the response across the receptive field. The average response across the receptive field for the different nociceptors in this study increased monotonically with stimulus force (Fig. 6). The highest intensity used in the present study was at the threshold for pain according to a psychophysical study that used a similar blade probe (Huang et al., 2000). We did not use higher stimulus intensities in this study to avoid damaging the skin. Whether stimulus-response functions would continue to increase with more intense stimuli needs to be determined.

As shown in Figure 7, with each doubling in force, the A-fiber nociceptors had a quadrupling in discharge, whereas the C-fibers only had a doubling in discharge. This was true over the entire force range $(40-640 \mathrm{mN})$. In previous studies in which stimuli were confined to the hot spot, A-fiber nociceptors also had steeper stimulus-response functions than C-fiber nociceptors. However, in those studies, the responses in the C-fibers approached a plateau with only one or two doublings in force.

In conclusion, the spatial mapping of responses of nociceptors resurrects the hypothesis that $\mathrm{C}$ - and A-fiber mechanically sensitive nociceptors could indeed play a role in signaling the pain from sharp mechanical stimuli. Future studies will be necessary with more substantial forces and with attendant psychophysical correlations to test this hypothesis. In addition, the neural coding of long-duration mechanical stimuli (Andrew and Greenspan, 1999; Schmidt et al., 2000) and impact stimuli (Koltzenburg and Handwerker, 1994) likely differ from those reported here for short-duration constant-force stimuli. The population-response analysis performed in this study is applicable to these noxious stimuli as well as to other stimuli that activate nociceptors such as intense heat and noxious chemical stimuli. 


\section{References}

Andrew D, Greenspan JD (1999) Peripheral coding of tonic mechanical cutaneous pain: comparison of nociceptor activity in rat and human psychophysics. J Neurophysiol 82:2641-2648.

Campbell JN, Meyer RA (1983) Sensitization of unmyelinated nociceptive afferents in the monkey varies with skin type. J Neurophysiol 49:98-110.

Garell PC, McGillis SLB, Greenspan JD (1996) Mechanical response properties of nociceptors innervating feline hairy skin. J Neurophysiol 75:1177-1189.

Greenspan JD, McGillis SLB (1991) Stimulus features relevant to the perception of sharpness and mechanically evoked cutaneous pain. Somatosens Motor Res 8:137-147.

Huang JH, Ali Z, Travison TG, Campbell JN, Meyer RA (2000) Spatial mapping of the zone of secondary hyperalgesia reveals a gradual decline of pain with distance but sharp borders. Pain 86:33-42.

Johnson KO, Lamb GD (1981) Neural mechanisms of spatial tactile discrimination: neural patterns evoked by braille-like dot patterns in the monkey. J Physiol (Lond) 310:117-144.

Julius D, Basbaum AI (2001) Molecular mechanisms of nociception. Nature 413:203-210.

Kenins P (1988) The functional anatomy of the receptive fields of rabbit C polymodal nociceptors. J Neurophysiol 59:1098-1115.

Khalsa PS, LaMotte RH, Grigg P (1997) Tensile and compressive responses of nociceptors in rat hairy skin. J Neurophysiol 78:492-505.

Khalsa PS, Zhang C, Qin YX (2000) Encoding of location and intensity of noxious indentation into rat skin by spatial populations of cutaneous mechano-nociceptors. J Neurophysiol 83:3049-3061.

Koltzenburg M, Handwerker HO (1994) Differential ability of human cutaneous nociceptors to signal mechanical pain and to produce vasodilatation. J Neurosci 14:1756-1765.
Peng YB, Ringkamp M, Meyer RA, Campbell J (2003) Fatigue and paradoxical enhancement of heat response in C-fiber nociceptors from crossmodal excitation. J Neurosci 23:4766-4774.

Raja SN, Meyer RA, Ringkamp M, Campbell JN (1999) Peripheral neural mechanisms of nociception. In: Textbook of pain (Wall PD, Melzack P, eds), pp 11-57. Edinburgh: Churchill.

Schmidt R, Schmelz M, Torebjörk HE, Handwerker HO (2000) Mechanoinsensitive nociceptors encode pain evoked by tonic pressure to human skin. Neuroscience 98:793-800.

Schneider W, Slugg RM, Turnquist BP, Meyer RA, Campbell JN (1995) An electromechanical stimulator system for neurophysiological and psychophysical studies of pain. J Neurosci Methods 60:61-68.

Slugg RM, Meyer RA, Campbell JN (2000) Response of cutaneous A- and C-fiber nociceptors in the monkey to controlled-force stimuli. J Neurophysiol 83:2179-2191.

Tillman DB (1992) Heat response properties of unmyelinated nociceptors. $\mathrm{PhD}$ thesis, The Johns Hopkins University.

Tillman DB, Treede R-D, Meyer RA, Campbell JN (1995) Response of C fibre nociceptors in the anaesthetized monkey to heat stimuli: estimates of receptor depth and threshold. J Physiol (Lond) 485 3:753-765.

Treede R-D, Meyer RA, Campbell JN (1998) Myelinated mechanically insensitive afferents from monkey hairy skin: heat-response properties. J Neurophysiol 80:1082-1093.

Vallbo B, Olausson H, Wessberg J, Kakuda N (1995) Receptive field characteristics of tactile units with myelinated afferents in hairy skin of human subjects. J Physiol (Lond) 483:783-795.

Wessberg J, Olausson H, Fernstrm KW, Vallbo AB (2003) Receptive field properties of unmyelinated tactile afferents in the human skin. J Neurophysiol 89:1567-1575. 